\title{
Estimation of the structural heterogeneity of Tick-Borne Encephalitis vaccine particles
}

Andrey Moiseenko ${ }^{1}$, Petr Zaitsev ${ }^{2}$, Mikhail Vorovitch ${ }^{3}$, Dmitry Osolodkin ${ }^{4}$, Aidar Ishmukhametov ${ }^{3}$, Alexey Egorov $^{3}$, Konstantin Shaitan ${ }^{2}$, Mikhail Kirpichnikov ${ }^{5}$ and Olga Sokolova ${ }^{1}$

${ }^{1}$ Moscow Lomonosov University, United States, ${ }^{2}$ Moscow Lomonosov State University, United States,

${ }^{3}$ Federal State Budgetary Scientific Institution "Chumakov Federal Scientific Center for Research and Development of Immune-and-Biological Products of Russian Academy of Sciences, United States, ${ }^{4}$ Federal State Budgetary Scientific Institution "Chumakov Federal Scientific Center for Research and Development of Immune-and-Biological Products of Russian Academy of Sciences”, United States, ${ }^{5}$ Faculty of Biology, Lomonosov Moscow State University, 119234 Moscow, Russia., United States

\section{Introduction}

Tick-borne encephalitis (TBE) is a severe infectious disease caused by the tick-borne encephalitis virus (TBEV), a typical member of the Flavivirus genus of the Flaviviridae family. Inactivated vaccines are widely used to prevent thisdisease [1]. The structure of the viral particles forming the vaccine antigen was never studied in detail sufficient enough to characterize it on the atomic level. Yet, such information is important for the comparison of the envelope protein E epitopes in the inactivated preparations and the wild-type virions, eventually useful for the rational structure-based vaccine and antibody design. Here we present an approach based on the use of analytical electron microscopy (EM) to quantify the heterogeneity of TBEV particles in purified samples for further single-particle imaging (SPI) on X-ray free electron lasers (XFEL), which provide a unique opportunity to study the time-resolved solution structures of large macromolecular assemblies, such as viruses. The requirements for a virus sample for SPI study at XFELs include high concentration of particles, knowledge of particle size distribution, and absence of aggregates [2]. We established a methodological workflow for such characterization of inactivated TBEV samples.

\section{Methods}

The TBEV (strain Sofjin, Genbank KC806252 [3]) was propagated in primary chicken embryo fibroblast culture. The purification protocol was based on tangential flow filtration and two steps of ultracentrifugation, one of them through the sucrose gradient. Viral particles in the resulting samples were quantified by ELISA. The concentration of the sample was $\sim 10^{13} \mathrm{~mL}^{-1}$.

Electron Microscopy was performed with a JEOL JEM-2100 200kV LaB6 transmission electron microscope equipped with a Gatan GIF Quantum ER Electron Energy Loss (EEL) spectrometer, along with a HAADF (High-Angle Annular Dark-Field) detector. The JEOL 21090 cooling holder was operated at $-180^{\circ} \mathrm{C}$ to reduce the contamination effects and to enhance the specimen's stability under the electron beam. The microscope was operated in STEM mode with a $5 \mathrm{~nm}$ probe size and a 6 mrad spectrometer collection angle. Spectrum data for elemental mapping were collected with $0,25 \mathrm{eV} / \mathrm{channel}$ energy dispersion with 2048 detector channels. The spectrum data were aligned by carbon $\mathrm{K}$ peak at $283 \mathrm{eV}$. The background was extrapolated with power law over a 109-128 eV window, the signal window for phosphorus mapping was set to a 132-155 eV phosphorus L2,3 peak range. No multiple scattering correction was applied. 


\section{Results}

We studied the inactivated TBEV particles using negative-stain EM (Fig. 1A). For the size analysis, we used 2\% uranyl-acetate stain. We collected 3606 single particles and measured their overall size. The spherical particles possess an average diameter of $46 \mathrm{~nm}$ (Fig. 1B) with dispersion from 40 till $54 \mathrm{~nm}$. This variation reflects the virion heterogeneity, attributable to different states of the particle maturity and completeness, as well as the conformational mobility.

We developed an analytical approach to evaluate the heterogeneity of the nucleocapsids. For this, we used negative stain with $2 \%\left(\mathrm{NH}_{4}\right)_{2} \mathrm{MoO}_{4}$, since the Uranium peak interferes with the Phosphorous peak in EELS. The distribution of RNA in the viral capsid was estimated by EELS, using the Phosphorus signal. The Phosphorous (P) signal was mapped onto the virion images after background subtraction and multiple scattering correction with the Fourier-ratio deconvolution. (Fig. 2). All investigated virions contain the Phosphorus signal, but its spatial distribution and intensity differ significantly (Fig. 2B). This clearly reflects the non-even distribution of RNA inside the nucleocapsid, which, apparently, is a reason for the heterogeneity of the TBEV particles, which apparently accompanies their structural heterogeneity, previously observed by cryo-electron microscopy [4].

This work was supported by RFBR grants \#18-02-40010 and \# 18-02-40026. Analytical microscopy was performed at the Unique equipment setup "3D-EMC” of Moscow State University, Biology Department.
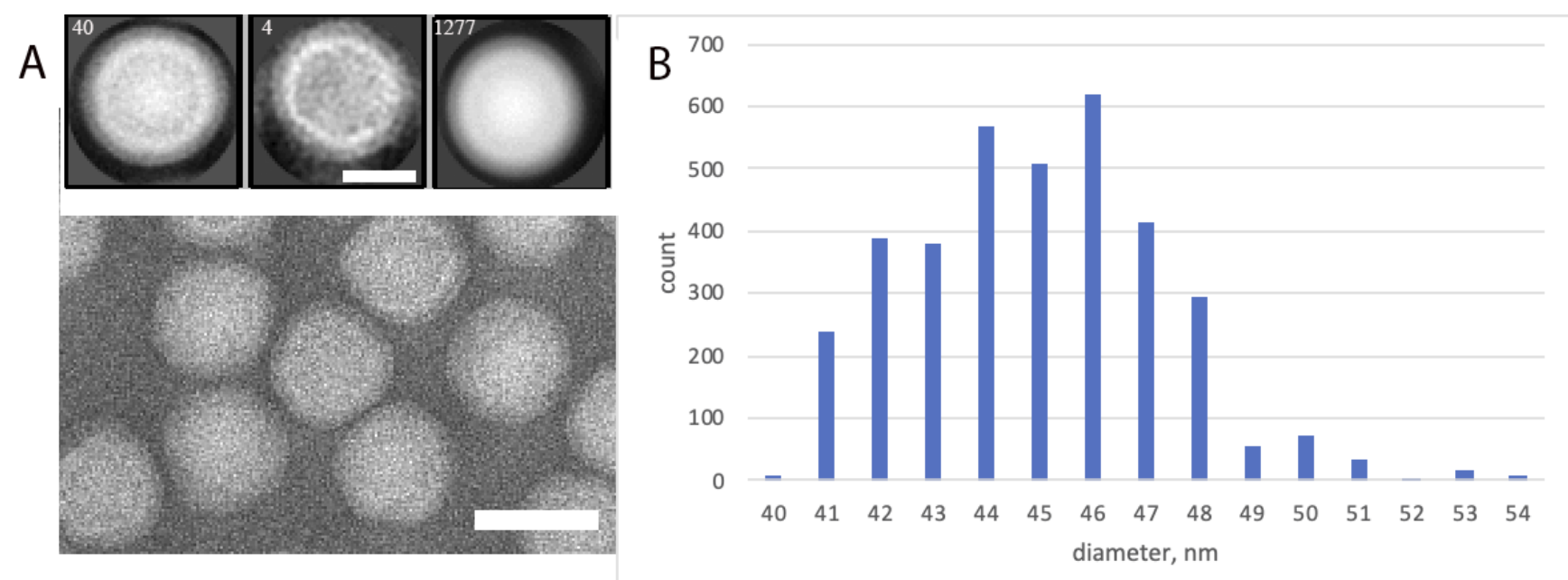

Figure 1. Figure 1. Image processing of TBEV particles. (A) Top - class-averages. Bar size: $25 \mathrm{~nm}$. Digits reflect number of particles in each class; bottom - raw TBEV particles. Negative stain with $2 \%$ uranylacetate. Bar size: $50 \mathrm{~nm}$; (B) Plot of particle diameters. 

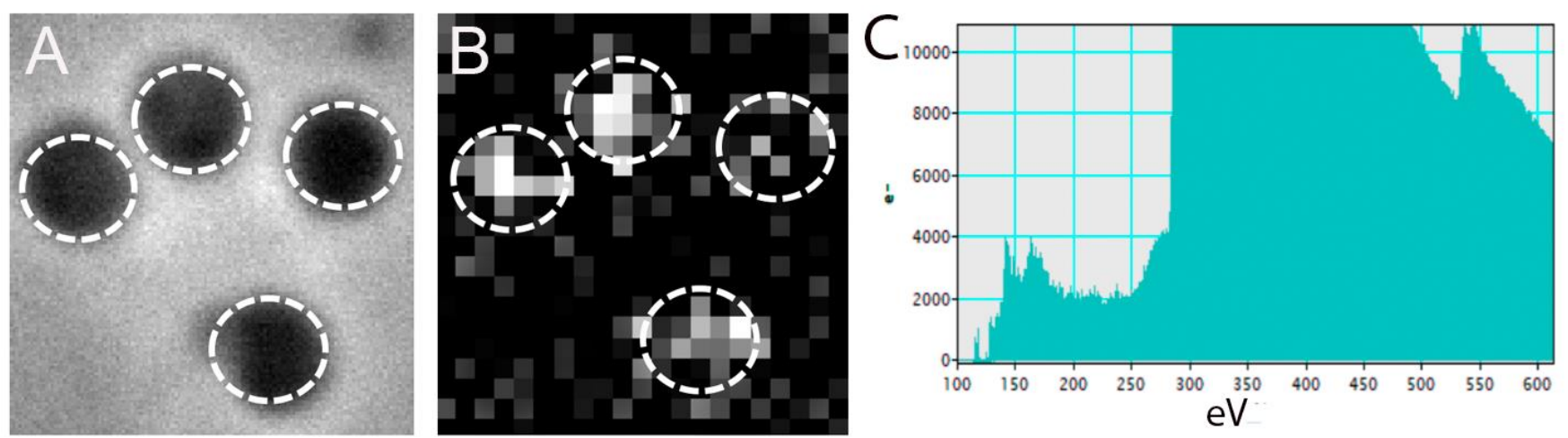

Figure 2. Figure 2 Analytical EM of Phosphorus distribution inside TBEV particles. (A) HAADF spectrum of four viral particles. Negative stain with 2\% (NH4)2MoO4; (B) Phosphorus mapping over the same areas, as in (A). Different shadows of white reflect different concentration of $P$. White dashed circles show the borders of TBEV particles. (C) The EELS spectrum data, reflecting the P peak.

\section{References}

1. Ruzek D, Avšič Županc T, Borde J, Chrdle A, Eyer L, Karganova G, Kholodilov I, Knap N, Kozlovskaya L, Matveev A et al. Tick-borne encephalitis in Europe and Russia: Review of pathogenesis, clinical features, therapy, and vaccines. Antiviral Res. 2019; 164:23-51. doi:10.1016/j.antiviral.2019.01.014. PMID: 30710567.

2. Anton Y. Fedotov, Mikhail F. Vorovitch, Kseniya K. Tuchinskaya, Konstantin V. Grishin, Olga I. Konyushko1, Dmitry I. Osolodkin, Aidar A. Ishmukhametov, Alexey M. Egorov. Abstract P-28: Protocol of Tick-Borne Encephalitis Virus Sample Preparation for Structural Studies. INTERNATIONAL JOURNAL OF BIOMEDICINE. 2019; v. 9, № S16 p. 29.

3. Vorovitch MF, Kozlovskaya LI, Romanova LIu, Chernokhaeva LL, Ishmukhametov AA, Karganova GG. Genetic description of a tick-borne encephalitis virus strain Sofjin with the longest history as a vaccine strain. Springerplus. 2015; 4: 761. doi: 10.1186/s40064-015-1561-y. PMID: 26682114.

4. Samygina VR, Pichkur EB, Vorovich MF, Konarev PV, Peters GG, Osolodkin DI, Ishmukhametov AA, Egorov AM. Abstract P-29: Quality Control of Tick-Bone Encephalitis Virus Samples Using TEM and SAXS for XFEL Studies. Int J Biomed. 2019; 9(S1):29-30. doi: 10.21103/IJBM.9.Suppl_1.P29. 